\title{
Efficient Calculation of Sensitivity Measures for Haldane Model Equations
}

\author{
G.S.C. Okpokwasili ${ }^{1}$, E.N. Ekaka-a ${ }^{1}$, C.O. Nweke $^{2}$ \\ ${ }^{I}$ Department of Microbiology, University of Port Harcourt, P.M.B. 5323, Port Harcourt, Nigeria \\ ${ }^{1}$ Department of Computer Science and Mathematics, Rivers State University of Science and Technology, Port \\ Harcourt, Nigeria, \\ ${ }^{2}$ Department of Microbiology, Federal University of Technology, Owerri, Nigeria
}

\begin{abstract}
The application of kinetic modelling of toxicity, adsorption and biodegradation of phenolic compounds is an active area of research which uses the tool of mathematical modelling to understand the complex interaction of substrate depletion and biomass production. However, it is imperative to test the sensitivity of the model parameters which define these key processes of applied microbiology in order to find out which of these parameters will have either a biggest or smallest cumulative effect on the model output or solution trajectory. Since sensitivity analysis is an integral part of model development and is capable of providing useful insights for a further validation research in applied biodegradation of phenolic compounds, it is a challenging collaborative scientific investigation to attempt to find which model parameters of this microbiological system would require additional research for the purpose of strengthening the continuity of knowledge base thereby reducing output uncertainty.

In this numerical study, we have used the technique of sensitivity measures or sensitivity analysis to select the maximum specific growth rate, the experimental time and the starting substrate value as the relatively sensitive parameters while other model parameters can be classified as relatively least sensitive. We will expect these contributions to guide further research in the validation of Monod models of substrate depletion and biomass production.
\end{abstract}

Key words: Microbial Growth, Substrate Concentration, Kinetics, Monod Model, Sensitivity Measures

\section{Introduction}

It is a well-established fact that toxicity, adsorption and biodegradation of phenolic compounds depends on the complex process of mathematical modelling which forms the basis of Okpokwasili and Nweke model formulation [2005] being an extension of Monod's model. However, the sensitivity measures of the parameters which define the dynamics of this interaction were yet to be empirically determined. The sensitivity of one model parameter over another model parameter is an integral part of model development which can be used to guide further research and strengthen knowledge-base in this challenging interdisciplinary theme of the kinetic modelling of toxicity, adsorption and biodegradation of phenolic compounds. It is against this background that we intend for the first time to determine the sensitivity measures of a Monod's model with the expectation of providing some insights which we have not seen elsewhere on an aspect of the kinetic modelling of toxicity, adsorption and biodegradation of phenolic compounds.

\section{Materials and Methods}

According to Nweke (2010), the observations which were made on phenol inhibition of growth of culture and biodegradation can be modelled using pioneering substrate inhibition models which have already been developed and tested experimentally by Okpokwasili and Nweke, 2005. On the basis of this formulation, the Haldane model 1 is defined by the following first order ordinary differential equation which describes substrate depletion $\mathrm{dS} / \mathrm{dt}=-\mu_{\mathrm{m}} \mathrm{S}\left(\mathrm{YS}_{0}-\mathrm{YS}+\mathrm{X}_{0}\right) /\left(\mathrm{K}_{\mathrm{s}}+\mathrm{S}\right)\left(1+\mathrm{S} / \mathrm{K}_{\mathrm{i}}\right) \mathrm{Y}$

on the assumption that the starting substrate concentration is defined as a positive constant $S(0)$ while the parameters $\mu_{\mathrm{m}}, \mathrm{Y}, \mathrm{S}_{0}, \mathrm{X}_{0}$ and $\mathrm{K}_{\mathrm{s}}$ are positive constants. Here, the dependent variable $\mathrm{S}$ stands for the substrate concentration, the parameter $\mu_{\mathrm{m}}$ stands for the maximum specific growth rate, $\mathrm{K}_{\mathrm{s}}$ stands for the substrate saturation constant, that is, substrate concentration at half $\mu_{\max }$ and $\mathrm{Y}$ is called the true growth yield [mass of biomass (X) synthesized per unit of substrate (S) utilized or removed]. The Haldane model 2 is similarly defined by the equation

$\mathrm{dS} / \mathrm{dt}=-\mu_{\mathrm{m}} \mathrm{S}\left(\mathrm{YS}_{0}-\mathrm{YS}+\mathrm{X}_{0}\right) /\left(\mathrm{K}_{\mathrm{s}}+\mathrm{S}+\mathrm{S}^{2} / \mathrm{K}_{\mathrm{i}}\right) \mathrm{Y}$

In this study, we propose to use the method of sensitivity measures or sensitivity analysis to find which parameters when varied a little one-at-a-time will produce either the biggest cumulative effect or smallest 
cumulative effect on the model output or solution trajectory. This numerical idea of tackling important open scientific problems in parameter estimation and model validation has already been defined clearly and applied in the recent published research reports of Ekaka-a and Nafo [2012a, 2012b] which complements other scientific problems of different patterns of formulation and sophistication [Gardner et al., 1980; O' Neill et al., 1980; Downing et al., 1985; Breshears, 1987; Crick et al., 1987; Yu et al., 1991; Ekaka-a, 2009].

When a chosen model parameter is varied a little while other parameters are fixed, we have used the three popular mathematical norms which measure the error between the data points of original substrate depletion model and the simulated substrate depletion model due to a variation of a model parameter to calculate the cumulative percentage effect on the model output under a fixed length of experimental time in a maximum of 10 hours.

How do we calculate the sensitivity measures for each model parameter? The Haldane model 1 has six parameters namely $\mu_{\mathrm{m}}, \mathrm{Y}, \mathrm{K}_{\mathrm{s}}, \mathrm{S}_{0}, \mathrm{X}_{0}$, and $\mathrm{K}_{\mathrm{i}}$. To illustrate this proposed numerical technique, consider the parameter $\mu_{\mathrm{m}}$. The chosen precise value of $\mu_{\mathrm{m}}$ is 0.01 . First, we vary this parameter by $10 \%$ and measure its cumulative percentage effect on the solution trajectory or model output. The solution trajectory when this parameter is not varied and the solution trajectory when the same parameter is varied behave differently. It is expected that the difference of these two solution trajectories over time will incur some numerical error. This error can be quantified by using the three popular mathematical norms of 1-norm, 2-norm, and infinity norm. The numerical values of these norms are calculated by dividing the norm of the difference of the solution trajectories when the parameter is varied by the norm of the difference of the solution trajectories when the parameter is not varied. The output of this calculation is quantified in terms of percentage. We observe that in one instance, the 1-norm value of the solution trajectories may be bigger than the 2-norm value of the same solution trajectories while the 2-norm value of the same solution trajectories is bigger than the infinity norm of the solution trajectories. We follow the same procedure to calculate the sensitivity measures of parameter $\mu_{\mathrm{m}}$ due to other variations such as $12 \%, 14 \%, 16 \%$, and $18 \%$. In this scenario and other scenarios which we have reported below, we observe that the values of the mathematical norms all decrease from the $10 \%$ parameter value variation to $18 \%$ parameter value variation. Because of the challenge and limitation of the analytical calculation in terms of computational time and the degree of correctness of the sensitivity values, we have adopted to utilize the ODE 45 [Ordinary Differential Equations of Order 4-5 due to Runge-Kutta Scheme] sensitivity technique in this present analysis. We opted to implement only the ODE 45 sensitivity technique over the ODE 23 [Ordinary Differential Equations of Order 2-3 due to Runge-Kutta Scheme] sensitivity technique because these two numerical techniques provide similar cumulative percentage effects on the solution trajectories. The numerical ODE 45 sensitivity technique is preferred in this context because it is computationally efficient than the ODE 23 technique. The results which we have obtained using this powerful numerical technique of sensitivity analysis are reported and discussed in the next section of this paper.

\section{Results for Haldane Model 1}

In this section, we will presentthe results which we have obtained on applying sensitivity analysis to the individual parameters of the substrate depletion Haldane model 1 formulation.

\begin{tabular}{|l|l|l|l|l|l|}
\hline \multirow{2}{*}{$\begin{array}{l}\text { Each Type of Mathematical } \\
\text { Norms }\end{array}$} & \multicolumn{5}{|c|}{ Variations of $\mu_{\mathrm{m}}=0.01$} \\
\cline { 2 - 6 } & 0.001 & 0.0012 & 0.0014 & 0.0016 & 0.0018 \\
\hline 1-norm & 327.93 & 265.40 & 220.66 & 187.33 & 161.30 \\
\hline 2-norm & 146.46 & 130.80 & 118.41 & 108.18 & 99.64 \\
\hline Infinity-norm & 88.74 & 86.40 & 84.01 & 81.58 & 79.12 \\
\hline
\end{tabular}

Table 1: Sensitivity Measures of Parameter $\mu_{\mathrm{m}}$

What do we learn from Table 1? When the parameter $\mu_{\mathrm{m}}$ is varied by 10 percent, its new value is 0.001 . When parameter $\mu_{\mathrm{m}}$ takes a precise value of 0.001 , this change produces 327.93 cumulative percentage change on the model output or solution trajectories using a 1-norm sensitvity method. Similarly, for this 10 percentage variation of parameter $\mu_{\mathrm{m}}$, the 2-norm and infinity-norm sensitivity methods will produce 146.46 and 88.74 cumulative percentage changes on the solution trajectories. On the other hand, when the same model parameter is varied by 18 percent, the three sensitvity values of 1-norm, 2-norm and infinity-norm are 161.30, 99.64, and 79.12 cumulative percentage changes. On the basis of this systematic analysis, the model parameter $\mu_{\max }$ can be classified as a most sensitively parameter. Comparable numerical analyses have been conducted for other model parameters which we have displayed our contributions in Table 2, Table 3, Table 4, Table 5, and Table 6 . Within these percentage variations of $\mu_{\mathrm{m}}$, we observe that the sensitivity values unanimously decrease from a high value of sensitivity to a relatively high value of sensitivity irrespective of the mathematical norms which were utilized to calculate the sensitivity values. The weighted mean which corresponds to the 1-norm, 2-norm, and infinity norm sensitivity values can be statistically determined because each new value of the parameter $\mu_{\mathrm{m}}$ 
has specific proportion in terms of the calculated 1-norm, 2-norm, and infinity norm. We decided to omit this calculation because it is not the focus of our research.

In the subsequent analyses, we have followed the same method of calculating the ODE 45 sensitivity values when the model parameters $\mathrm{Y}, \mathrm{K}_{\mathrm{s}}, \mathrm{S}_{0}, \mathrm{X}_{0}$, and $\mathrm{K}_{\mathrm{i}}$ are varied from $10 \%, 12 \%, 14 \%, 16 \%$ to $18 \%$. The corresponding sensitivity results which we have obtained due to these variations are also clearly presented and displayed in the Tables below.

\begin{tabular}{|l|l|l|l|l|l|}
\hline \multirow{2}{*}{$\begin{array}{l}\text { Each Type of Mathematical } \\
\text { Norms }\end{array}$} & \multicolumn{5}{|c|}{ Variations of Y $=0.001$} \\
\cline { 2 - 6 } 1-norm & 0.0001 & 0.00012 & 0.00014 & 0.00016 & 0.00018 \\
\hline 2-norm & 0.4492 & 0.4389 & 0.4424 & 0.4650 & 0.4441 \\
\hline Infinity-norm & 0.0239 & 0.0235 & 0.0237 & 0.0246 & 0.0236 \\
\hline
\end{tabular}

Table2: Sensitivity Measures of Parameter Y

\begin{tabular}{|l|l|l|l|l|l|}
\hline Each Type of Mathematical & \multicolumn{5}{|c|}{ Variations of $\mathrm{K}_{\mathrm{s}}=0.01$} \\
\cline { 2 - 6 } Norms & 0.001 & 0.0012 & 0.0014 & 0.0016 & 0.0018 \\
\hline 1-norm & 0.4177 & 0.4039 & 0.3867 & 0.3835 & 0.4078 \\
\hline 2-norm & 0.0228 & 0.0218 & 0.0206 & 0.0207 & 0.0217 \\
\hline Infinity-norm & 0.0032 & 0.0031 & 0.0030 & 0.0028 & 0.0027 \\
\hline
\end{tabular}

Table3: Sensitivity Measures of $\mathrm{K}_{\mathrm{s}}$

\begin{tabular}{|l|l|l|l|l|l|}
\hline \multirow{2}{*}{$\begin{array}{l}\text { Each Type of } \\
\text { Mathematical Norms }\end{array}$} & \multicolumn{5}{|c|}{ Variations of $\mathrm{S}_{0}=0.5$} \\
\cline { 2 - 6 } & 0.05 & 0.06 & 0.07 & 0.08 & 0.09 \\
\hline 1-norm & 0.4266 & 0.4439 & 0.4491 & 0.4416 & 0.4356 \\
\hline 2-norm & 0.0236 & 0.0240 & 0.0240 & 0.0241 & 0.0234 \\
\hline Infinity-norm & 0.0035 & 0.0032 & 0.0037 & 0.0036 & 0.0036 \\
\hline
\end{tabular}

Table4: Sensitivity Measures of $\mathrm{S}_{0}$

\begin{tabular}{|l|l|l|l|l|l|}
\hline \multirow{2}{*}{$\begin{array}{l}\text { Each Type of } \\
\text { Mathematical Norms }\end{array}$} & \multicolumn{5}{|c|}{ Variations of parameter $\mathrm{X}_{0}=0.03$} \\
\cline { 2 - 6 } 1-norm & 0.003 & 0.0036 & 0.0042 & 0.0048 & 0.0054 \\
\hline 2-norm & 283.17 & 234.30 & 198.25 & 169.97 & 148.42 \\
\hline Infinity-norm & 135.53 & 122.38 & 111.64 & 102.70 & 94.92 \\
\hline
\end{tabular}

Table 5: Sensitivity Measures of Parameter $\mathrm{X}_{0}$

\begin{tabular}{|l|l|l|l|l|l|}
\hline \multirow{2}{*}{$\begin{array}{l}\text { Each Type of Mathematical } \\
\text { Norms }\end{array}$} & \multicolumn{5}{|c|}{ Variations of parameter $\mathrm{K}_{\mathrm{i}}=0.02$} \\
\cline { 2 - 7 } & 0.0020 & 0.0024 & 0.0028 & 0.0032 & 0.0036 \\
\hline 1-norm & 211.30 & 171.59 & 139.17 & 118.54 & 105.30 \\
\hline 2-norm & 118.64 & 105.82 & 94.70 & 86.90 & 79.43 \\
\hline Infinity-norm & 85.05 & 84.86 & 78.78 & 75.57 & 72.30 \\
\hline
\end{tabular}

Table 6: Sensitivity Measures of Parameter $\mathrm{K}_{\mathrm{i}}$

\section{Results for Haldane Model 2}

The sensitivity results which we have obtained with respect to the Haldane model 2 will be presented in this section.

\begin{tabular}{|c|c|c|c|c|c|}
\hline \multirow{2}{*}{$\begin{array}{c}\text { Each Type of Mathematical } \\
\text { Norms }\end{array}$} & \multicolumn{5}{|c|}{ Variations of $\mu_{\mathrm{m}}=0.01$} \\
\cline { 2 - 6 } & 0.001 & 0.0012 & 0.0014 & 0.0016 & 157.06 \\
\hline 1-norm & 278.95 & 224.54 & 185.70 & 134.13 \\
\hline 2-norm & 134.87 & 120.00 & 108.17 & 98.46 & 90.11 \\
\hline Infinity-norm & 87.28 & 84.58 & 81.84 & 79.04 & 76.17 \\
\hline
\end{tabular}

Table 7: Sensitivity Measures of Parameter $\mu_{\mathrm{m}}$

\begin{tabular}{|c|l|l|l|l|l|}
\hline \multirow{2}{*}{$\begin{array}{c}\text { Each Type of Mathematical } \\
\text { Norms }\end{array}$} & \multicolumn{5}{|c|}{ Variations of Y $=0.01$} \\
\cline { 2 - 7 } & 0.0001 & 0.00012 & 0.00014 & 0.00016 & 0.00018 \\
\hline 1-norm & 0.47 & 0.4637 & 0.4546 & 0.4355 & 0.4539 \\
\hline 2-norm & 0.025 & 0.0247 & 0.0245 & 0.0234 & 0.0240 \\
\hline Infinity-norm & 0.0034 & 0.0033 & 0.0038 & 0.0033 & 0.0032 \\
\hline
\end{tabular}

Table 8: Sensitivity Measures of Parameter Y

\begin{tabular}{|c|l|l|l|l|l|}
\hline \multirow{2}{*}{$\begin{array}{c}\text { Each Type of Mathematical } \\
\text { Norms }\end{array}$} & \multicolumn{5}{|c|}{ Variations of $\mathrm{K}_{\mathrm{s}}=0.01$} \\
\cline { 2 - 6 } & 0.001 & 0.0012 & 0.0014 & 0.0016 & 0.0018 \\
\hline 1-norm & 0.4207 & 0.4176 & 0.4066 & 0.4078 & 0.4210 \\
\hline 2-norm & 0.0225 & 0.0224 & 0.0217 & 0.0218 & 0.0227 \\
\hline Infinity-norm & 0.0027 & 0.0033 & 0.0030 & 0.0032 & 0.0031 \\
\hline
\end{tabular}

Table 9: Sensitivity Measures of Parameter $K_{s}$ 
Efficient Calculation of Sensitivity Measures for Haldane Model Equations

\begin{tabular}{|c|c|c|c|c|c|}
\hline \multirow{2}{*}{$\begin{array}{l}\text { Each Type of Mathematical } \\
\text { Norms }\end{array}$} & \multicolumn{5}{|c|}{ Variations of $\mathrm{S}_{0}=0.5$} \\
\cline { 2 - 6 } & 0.05 & 0.06 & 0.07 & 0.08 & 0.09 \\
\hline 1-norm & 0.4669 & 0.4695 & 0.4690 & 0.4626 & 0.4648 \\
\hline 2-norm & 0.0251 & 0.0248 & 0.0249 & 0.0250 & 0.0251 \\
\hline Infinity-norm & 0.0036 & 0.0031 & 0.0036 & 0.0034 & 0.0035 \\
\hline
\end{tabular}

Table 10: Sensitivity Measures of Parameter $\mathrm{S}_{0}$

\begin{tabular}{|c|c|c|c|c|c|}
\hline \multirow{2}{*}{$\begin{array}{l}\text { Each Type of Mathematical } \\
\text { Norms }\end{array}$} & \multicolumn{5}{|c|}{ Variations of $\mathrm{X}_{0}=0.03$} \\
\cline { 2 - 6 } & 0.003 & 0.0036 & 0.0042 & 0.0048 & 0.0054 \\
\hline 1-norm & 240.01 & 197.68 & 166.16 & 142.18 & 122.10 \\
\hline 2-norm & 124.50 & 111.95 & 101.80 & 93.05 & 85.75 \\
\hline Infinity-norm & 85.51 & 82.83 & 80.08 & 77.28 & 74.41 \\
\hline
\end{tabular}

Table 11: Sensitivity Measures of Parameter $\mathrm{X}_{0}$

\begin{tabular}{|c|c|c|c|c|c|}
\hline \multirow{2}{*}{$\begin{array}{l}\text { Each Type of Mathematical } \\
\text { Norms }\end{array}$} & \multicolumn{5}{|c|}{ Variations of parameter $\mathrm{K}_{\mathrm{i}}=0.02$} \\
\cline { 2 - 6 } & 0.0020 & 0.0024 & 0.0028 & 0.0032 & 0.0036 \\
\hline 1-norm & 164.77 & 127.40 & 110.50 & 88.81 & 67.23 \\
\hline 2-norm & 104.15 & 92.38 & 82.64 & 72.80 & 66.27 \\
\hline Infinity-norm & 82.21 & 78.40 & 74.47 & 70.42 & 66.27 \\
\hline
\end{tabular}

Table 12: Sensitivity Measures of Parameter $\mathrm{K}_{\mathrm{i}}$

\section{Discussion of Results}

It is very clear from these systematic sensitivity calculations of the parameters which define the Haldane model 1 and Haldane model 2 that all these parameters are not equally sensitive. Irrespective of the type of Haldane model, the sensitivity behaviour of the model parameters is differentiated into two distinct groups. The most sensitivity group concerns the model parameters $\mu_{\mathrm{m}}, \mathrm{X}_{0}$, and $\mathrm{K}_{\mathrm{i}}$ while the least sensitivity group concerns the model parameters $\mathrm{Y}, \mathrm{K}_{\mathrm{s}}$, and $\mathrm{S}_{0}$.

\section{Conclusion}

By using the numerical technique of sensitivity analysis, we have found that the model parameters $\mu_{\max }$ and the experimental time have greater influence on the model outputs. The next sensitive parameter is the substrate starting value. These three parameters which define the dynamics of the Monod's model need to be efficiently estimated in order to provide reliable model predictions and minimise model output uncertainty. On the other hand, the least sensitive model parameters $\delta_{0}, \mathrm{X}_{0}, \mathrm{~K}_{\mathrm{s}}$, y which also contribute to the development of the Monod's model need to be taken cautiously as rough estimates in contrast to the principle of parsimony. We would expect this present contribution to provide useful insights in the field of kinetic modelling of toxicity, adsorption and biodegradation of phenolic compounds. A further sensitivity ranking of the parameters of Monod's model which is different from its sensitivity analysis is yet to be attempted. We propose to look into this level of sophisticated analysis in our next study.

\section{References}

[1] Breshears, D.D.: 1987, Uncertainty and sensitivity analyses of simulated concentrations of radionuclides in milk. Fort Collins, CO: Colorado State University, MS Thesis, pp. 1-69.

[2] Crick, M.J., Hill, M.D. and Charles, D.: 1987, 'The Role of Sensitivity Analysis in Assessing Uncertainty. In: Proceedings of an NEA Workshop on Uncertainty Analysis for PerformanceAssessments of Radioactive Waste Disposal Systems, Paris, OECD, pp. 1-258.

[3] Ekaka-a E.N., Computational and Mathematical Modelling of Plant Species Interactions in a Harsh Climate, PhD Thesis, University of Liverpool and University of Chester, 2009.

[4] Ekaka-a, E.N. and Nafo,N.M: 2012, Parameter Ranking of Stock Market Dynamics: A Comparative Study of the Mathematical Models of Competition and Mutualistic Interactions, Scientia African, Vol. 11 (No. 1), June 2012, pp. 36-43.

[5] Gardner, R.H.: Huff, D.D., O'Neill, R.V., Mankin, J.B., Carney, J. and Jones, J.: 1980, 'Application of Error Analysis to a Marsh Hydrology Model', Water Resources Res. 16, 659-664.

[6] O'Neill, R.V., Gardner, R.H., and Mankin, J.B. 1980, 'Analysis of Parameter Error in a Nonlinear Model', Ecol. Modelling.8, $297-$ 311.

[7] Downing, D.J., Gardner, R.H., and Hoffman, EO.: 1985, 'An Examination of Response-Surface Methodologies for Uncertainty Analysis in Assessment Models', Technometrics. 27, 151-163. (See also Letter to the Editor, by R.G. Easterling and a rebuttal, Technometrics28, 91-93, 1986).

[8] Nweke (2010) PhD Thesis

[9] Okpokwasili, G.C and Nweke, C.O 2005, Microbial growth and substrate utilization kinetics, African Journal of Biotechnology, Vol. 5(4), pp. 305-317.

[10] Yu, C., Cheng, J-J., and Zielen, A-J.: 1991, 'Sensitivity Analysis of the RESRAD, a Dose Assessment Code,' Trans. Am. Nuc. Soc. $64 ; 73-74$. 